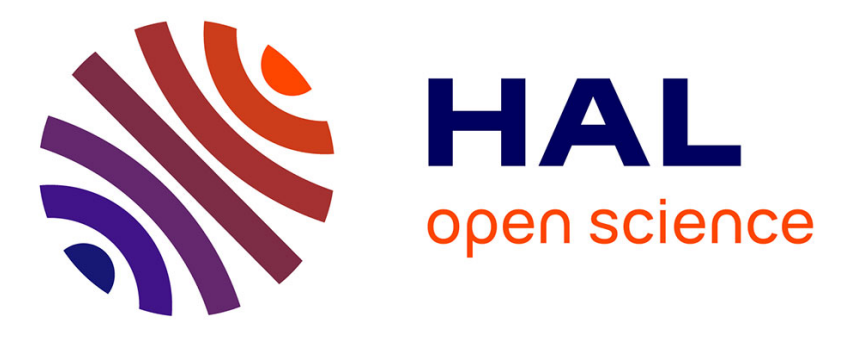

\title{
3D optical micro-resonators by curving nanostructures using intrinsic stress
}

Clément Sieutat, Céline Chevalier, Alexandre Danescu, Grenet Geneviève, Philippe Regreny, P. Viktorovich, Xavier Letartre, Jean-Louis Leclercq

\section{- To cite this version:}

Clément Sieutat, Céline Chevalier, Alexandre Danescu, Grenet Geneviève, Philippe Regreny, et al.. 3D optical micro-resonators by curving nanostructures using intrinsic stress. SPIE Photonics Europe 2012, Apr 2012, Bruxelles, Belgium. pp.8425-19, 10.1117/12.922375 . hal-00692513

\section{HAL Id: hal-00692513 https://hal.science/hal-00692513}

Submitted on 30 Apr 2012

HAL is a multi-disciplinary open access archive for the deposit and dissemination of scientific research documents, whether they are published or not. The documents may come from teaching and research institutions in France or abroad, or from public or private research centers.
L'archive ouverte pluridisciplinaire HAL, est destinée au dépôt et à la diffusion de documents scientifiques de niveau recherche, publiés ou non, émanant des établissements d'enseignement et de recherche français ou étrangers, des laboratoires publics ou privés. 


\title{
3D optical micro-resonators by curving nanostructures using intrinsic stress
}

\author{
C. Sieutat, C. Chevalier, A. Danescu, G. Grenet, P. Regreny, P. Viktorovitch, X. Letartre, J.L. \\ Leclercq \\ Université de Lyon, Institut des Nanotechnologies de Lyon (INL), UMR CNRS 5270 Ecole \\ Centrale de Lyon, 36 avenue Guy de Collongue, F 69134 Ecully Cedex, France
}

\begin{abstract}
We propose a new approach for the 3D control of light in real 3D optical micro-resonators that can be assimilated to 'cages', where photons are efficiently trapped. The main attractive feature of this photon cages lies in their ability to result in a considerable enhancement of the electromagnetic field in the central part of the cage, that is in the air region, opening the way to new sensing or trapping of nanoparticles in fluidic (gas or liquid) ambiances. Fabrication of three dimensional structures consists in exploiting the process of elastic relaxation of patterns formed in pre-stressed multi-layer structures. The final shape of these objects can be predetermined by the distribution of the deformations in the various semiconductor layers, imposed during their epitaxial growth, before their freestanding from the substrate by selective etching. We will present the basic concepts and fabrication we exploit to confine photons in air using spherical structures based on progressive relaxation of pre-stressed InGaP/InAsP bilayer films. It is worthwhile to notice that the formed microstructures exhibit patterns with dimensions compatible with optical operation in the visible/NIR wavelength range.
\end{abstract}

Keywords: 3D Photonic crystal, optical microcavity, microresonator, nanophotonics

\section{INTRODUCTION}

Today, materials exhibiting designed multidimensional architectures have extraordinary potential for emerging optical applications due to their ability to interact with light in ways that cannot be achieved using simple, non structured materials. Harnessing of light has always been central in the field of nanophotonics. This is usually referred to as photon confinement, whose general definition could be the control of photons within the tiniest possible space during the longest possible time. Fundamentally, the introduction of microstructure with characteristic dimensions similar to the wavelength of light can dramatically modulate light-matter interactions. Increasing dimensionality to two and three dimensions provides opportunities for far more exotic optical properties including 3D photonic bandgaps, metamaterials, optical cloaking, highly efficient solar cells, and unique sensors. 3D photonic crystals $(3 D P C)$ have attracted great interest because they likely provide a means of molding light at the level of physical limits. ${ }^{1}$ Only three dimensional photonic crystal can possess a complete photonic band gap, which potentially confines light in a small space due to omnidirectional inhibition of light propagation. The general approach to achieve strong confinement of photons consists in high index contrast structuring of space at the wavelength scale, which is in the submicrometer range for the optical domain. Proposed applications for photonic bandgap materials are quite diverse, and include lossless mirrors, the enhancement or inhibition of emission from embedded emitters, ${ }^{2}$ zero-threshold lasers, ${ }^{3}$ low-loss 3D micrometer-scale optical waveguides, ${ }^{4}$ optical circuitry, enhancement of optical absorption processes in solar cells, and sensors. ${ }^{5}$ Today, design and fabrication of multidimensional architectures for functional optical devices are of great interest, especially for bio and health applications (such as sensing, fluorescence imaging) where people are trying to strengthen the interactions with biological molecules or nanoparticles. For that, one needs to develop new strategies to confine light in air or, more generally, in low index materials like liquids or gas.

Fabrication technology of three-dimensional complete photonic bandgap materials in the optical domain is extremely complex. Two-dimensional photonic crystals (2D PC) are far more accessible than 3D PC, since they

Further author information: (Send correspondence to J.L.Leclercq)

J.L. Leclercq : E-mail: jean-louis.leclercq@ec-lyon.fr 
may be fabricated using planar technological schemes that are familiar to the world of integrated optics and microelectronics. Fabrication of three dimensional structures is then a significant challenge to be addressed. V.Y. Prinz et al. have first demonstrated such capabilities by fabricating nanotubes, nanowires and nanocoils using the strain relaxation of semiconductor layers. ${ }^{6}$ It was then considered as an emerging technology with a broad spectrum of possible applications. ${ }^{7}$ This technological approach combined with our 2.5D nanophotonics concept $^{8}$ yield us to design and create original 3 dimensional Micro-Nano-Photonics devices ('Optical Origami'). It is mainly a question of exploring and of combining new scientific and technological concepts founded on engineering of mechanical constraints to generate objects of various forms at the wavelength scale and to achieve a real 3D control of the light. In that purpose we have recently proposed a new concept of trapping photons in real 3D optical microresonators that can be assimilated to 'photon cages'. 9

The paper is organized as follows: the second section presents the basic principles and simulations associated to an "ideal" spherical object. The third section discusses about the basic mechanical ingredients needed for curvature and thickness control during fabrication, and presents numerical simulation of the folding problem for a "generic spiral object". In the fourth section we present the technological issues involved in the fabrication of three-dimensional objects and the design problem.

\section{PHOTON CAGE PRINCIPLES}

Novel class of highly efficient optical microcavities based on the $2.5 \mathrm{D}$ photonic crystals concept have been developed in our group. These are provided using ultra-thin and highly-efficient photonic crystal slab mirrors instead of conventional DBRs. A quasi-3D harnessing of the light at the wavelength scale are then obtained via the controllable interplay occurring between waveguided confined photons within the PC slabs and radiated photons propagating through the planar multilayer structure, at a far lower technological cost than pure $3 \mathrm{D}$ PC. ${ }^{10}$ 3D microcavities presenting closed geometries can be generated deviating from these ultimate photonic crystal mirrors-based vertical Fabry-Perot microcavities. These 'photon cages' can be seen as 'physical' deformation of the geometry of the vertical microcavity as shown schematically in Figure 1.

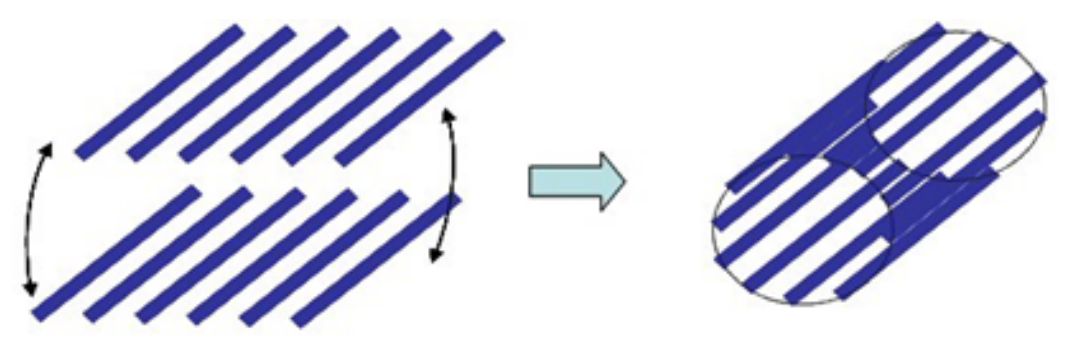

Figure 1. Schematic representation of the concept from $2.5 \mathrm{D}$ to $3 D$ microcavity. Left: vertical microcavity between 2 photonic crystal slab mirrors. Right: winding of this structure leading to a cylindrical microcavity.

By generating these micro-objects with various closed geometries (spires, tubes, rolls) at wavelength scales combined with dedicated optical modes engineering may yield to a tight spatial and spectral control of photons in the low index part (air region). Since these objects are mainly composed by air, their ability to sense or trap nanoparticles which can interfere with the cage resonant modes are drastically enhanced. In this article we focus on a specific 3D photonic structures which is a spherical cavity obtained by the relaxation of patterns formed in pre-stressed multi-layer structures (Figure 2).

\subsection{Simulating the electromagnetic properties of the $3 \mathrm{D}$ microstructure}

The electromagnetic properties of the three-dimensional photon cage were obtained using numerical simulation software developed at the Institut des Nanotechnologies de Lyon (Tessa). The software performs 3D FDTD simulations from an input file containing a discrete description of the refractive index. In order to build such a file, the coordinates of enough points on the 3D curve should be gathered, i.e., the sampling distance should be shorter than the space mesh. 


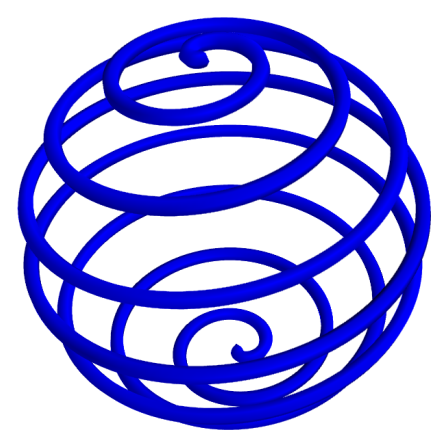

Figure 2. A general view of the 3D photonic spherical cavity

The parametric equation of the photon cage is given in (1), where $R$ is the radius and $N$ the number of turns in one half of a sphere $(-2 N \pi \leq \theta \leq 2 N \pi)$

$$
X(\theta)=R \sin \left(\frac{\pi}{2}-\frac{\theta}{4 N}\right) \cos (\theta), \quad Y(\theta)=R \sin \left(\frac{\pi}{2}-\frac{\theta}{4 N}\right) \sin (\theta), \quad Z(\theta)=R \cos \left(\frac{\pi}{2}-\frac{\theta}{4 N}\right)
$$

The curve itself is not sufficient to describe the photon cage and forms only the core of the structure. Depth and thickness around the curve must be added in such a way that they follow the progressive slope of the III-V SC material "ribbon" on each point of the curve. The orientation of the local Frenet-Serret frame can be obtained using the normal and binormal vectors (Figure 3). Let us consider a rectangle having the same dimensions as the section of this "ribbon". In order to obtain the 3D microstructure it can be centered on the curve and oriented using these vectors. With a sufficiently small step along the arc-length, the spherical photon cage representation can be realized (Figure 3). In addition this approach can be applied to build many other 3D microstructures starting from their parametric curve. In particular helical structures have been successfully designed using this method.
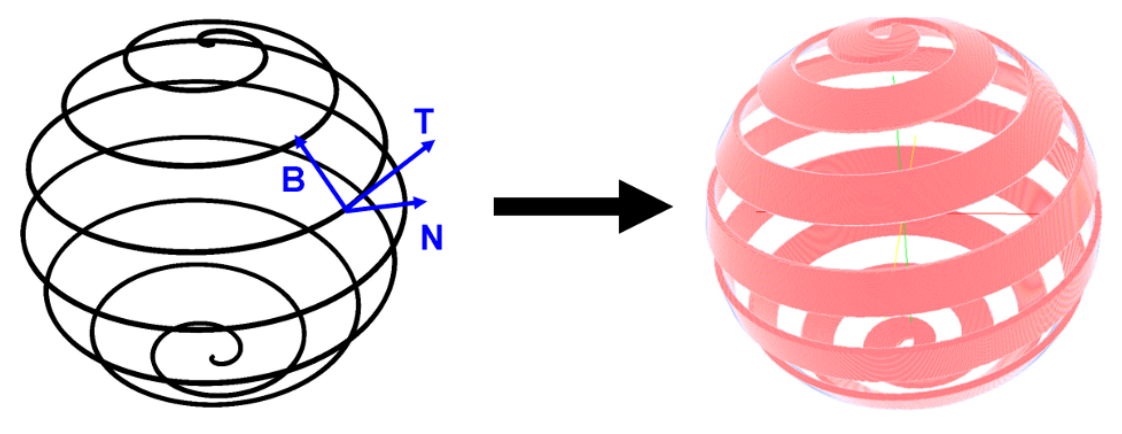

Figure 3. A Frenet-Serret base is represented of the parametric curve with tangent vector $\boldsymbol{T}$, normal vector $\boldsymbol{N}$ and binormal vector $\boldsymbol{B}$ (left). 3D view of the spherical photon cage (right).

An educated guess of the dimensions of the microstructure have been derived from a previously designed high reflectivity broadband $1 \mathrm{D} \mathrm{PhC}$ mirror similar to Figure $1 .{ }^{10}$ The thickness (along the binormal vector) is $0.40 \mu \mathrm{m}$ and the period along a longitudinal axis is $1.1 \mu \mathrm{m}$, leading to an approximate filling factor in material of 0.36. Finally the depth (along the normal vector) is $0.25 \mu \mathrm{m}$ and the sphere radius is $2.8 \mu m$. The electromagnetic properties of the spherical photon cage have been then determined by 3D FDTD simulations. A spatial step of $40 \mathrm{~nm}$ was chosen. The source consists in a dipole vibrating in the center of the cage. The value of the electromagnetic field is recorded on an off-center point after extinction of the source. A resonant mode is observed at $1.49 \mu \mathrm{m}$ with a quality factor of $Q=280$. It is worthwhile to notice the high field concentration reached at the center of the cage (Figure 4), although the cavity is composed mainly of air. Further tunings of the dimensions are expected to give modes with higher Q-factor. 


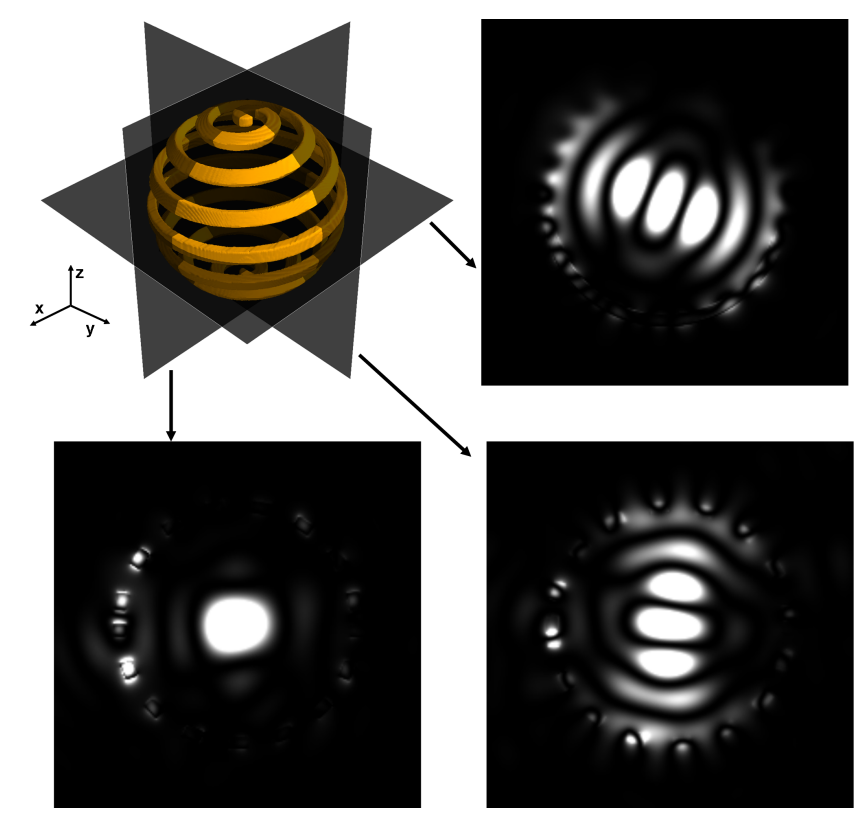

Figure 4. $E_{x}^{2}$ field profiles obtained by 3D FDTD on the three main axes: ( $Y Z$ ) plane (top right), (XZ) plane (bottom left), $(X Y)$ plane (bottom right).

In order to fabricate such structures we will use the relaxation of patterns formed in pre-stressed multi-layer semiconducting material. Then mechanical simulations are needed to design the flat shape that will give the final three-dimensional object (Figure 2) through the stress relaxation process.

\section{CURVATURE CONTROL AND DESIGN OF THREE-DIMENSIONAL OBJECTS}

This section presents the details of the mechanical side of the problem intended to : (i) discuss the dependence of a stress-free bilayer and delineate the compositions, thicknesses and misfit restricted by some technological constraints and (ii) the design problem relating the design of a two-dimensional mask to the shape of a stress-free three dimensional object.

\subsection{Curvature of the $\left(A, h_{A}\right) /\left(B, h_{B}\right)$ stress-free bilayer}

We consider an elastic layer $A$ (thickness $h_{A}$ ) grown on an elastic layer $B$ (thickness $h_{B}$ ). Let $m=\frac{a_{B}-a_{A}}{a_{A}}$ be the in-plane misfit between the $A$-layer and the $B$-layer and $\boldsymbol{C}^{A}$ and $\boldsymbol{C}^{B}$ denote the Hooke tensors of the layers. With respect to the stress-free reference configuration of the $B$ layer, the reduced constitutive law (Hooke law under plane stress assumptions) for the two materials are

- For the layer $A: \boldsymbol{\sigma}=\boldsymbol{C}^{A}[\boldsymbol{\varepsilon}+\boldsymbol{m}]$ where $\boldsymbol{m}=m \boldsymbol{I}_{2 \times 2}$.

- For the layer $B: \sigma=C^{B}[\varepsilon]$

As usual in classical plate theory the strain of the bilayer can be written as the sum between two terms:

$$
\varepsilon=\varepsilon^{0}+z \kappa
$$

where $\varepsilon^{0}$ and $\boldsymbol{\kappa}$ represent respectively the in-plane strain and curvature of the bilayer. In (2) the vertical coordinate $z$ is measured from the middle plan of the bilayer. The etching process renders the bilayer stress-free and thus induces both an in-plane strain and a curvature and the values of these can be obtained by imposing that the generalized efforts vanish. We obtain the system of equations

$$
\left(\begin{array}{cc}
\boldsymbol{A} & \boldsymbol{B} \\
\boldsymbol{B}^{t} & \boldsymbol{D}
\end{array}\right)\left(\begin{array}{c}
\varepsilon^{0} \\
\boldsymbol{\kappa}
\end{array}\right)=\left(\begin{array}{c}
\boldsymbol{N}^{0} \\
\boldsymbol{M}^{0}
\end{array}\right)
$$


where

$$
\boldsymbol{A}=h_{A} \boldsymbol{C}^{A}+h_{B} \boldsymbol{C}^{B}, \quad \boldsymbol{B}=\frac{1}{2} h_{A} h_{B}\left(\boldsymbol{C}^{A}-\boldsymbol{C}^{B}\right), \quad \boldsymbol{D}=\frac{1}{12}\left[h_{A}\left(3 h_{B}^{2}+h_{A}^{2}\right) \boldsymbol{C}^{A}+h_{B}\left(3 h_{A}^{2}+h_{B}^{2}\right) \boldsymbol{C}^{B}\right]
$$

and

$$
\boldsymbol{N}^{0}=h_{A} \boldsymbol{C}^{A}[\boldsymbol{m}], \quad \boldsymbol{M}^{0}=\frac{1}{12} h_{A} h_{B} \boldsymbol{C}^{A}[\boldsymbol{m}] .
$$

\subsubsection{Solution in the general case with cubic symmetry}

If both materials $A$ and $B$ have cubic symmetry it follows that $\varepsilon_{12}^{0}=\kappa_{12}^{0}=0$. Also from symmetry considerations we obtain $\varepsilon_{11}^{0}=\varepsilon_{22}^{0}$ and we denote their common value by $\varepsilon^{0}$. A similar result holds for the curvature where $\kappa_{11}=\kappa_{22}$ so that we use $\kappa$ for their common value. If we denote the plan modulus $Y_{A, B}=C_{11}^{A, B}+C_{12}^{A, B}=\frac{E^{A, B}}{1-\nu^{A, B}}$ the system (3) reduces to the two equations for $\varepsilon^{0}$ and $\kappa$ in the form

$$
\left\{\begin{array}{l}
\left(h_{A} Y_{A}+h_{B} Y_{B}\right) \varepsilon^{0}+\frac{1}{2} h_{A} h_{B}\left(Y_{A}-Y_{B}\right) \kappa=-m h_{A} Y_{A} \\
\frac{1}{2} h_{A} h_{B}\left(Y_{A}-Y_{B}\right) \varepsilon^{0}+\frac{1}{12}\left[h_{B}\left(3 h_{A}^{2}+h_{B}^{2}\right) Y_{B}+h_{A}\left(3 h_{B}^{2}+h_{A}^{2}\right) Y_{A}\right] \kappa=-\frac{1}{2} h_{A} h_{B} Y_{A} m .
\end{array}\right.
$$

In the following it is convenient to introduce the contrast $C=Y_{A} / Y_{B}$ and the thickness ratio $\xi=h_{A} / h_{B}$. The system (6) becomes

$$
\left\{\begin{array}{l}
(1+\xi C) \varepsilon^{0}+\frac{1}{2}(1-C) \kappa=-m \\
(1-C) \varepsilon^{0}+\frac{1}{6}\left[\left(3 \xi+\frac{1}{\xi}\right) C+3+\xi^{2}\right] \hat{\kappa}=-m .
\end{array}\right.
$$

where $\hat{\kappa}$ denotes $h_{B} \kappa$. Finally, the curvature of the bilayer is given by

$$
\kappa=-\times \frac{C \xi(1+\xi)}{\xi^{3}+C^{2} \xi\left(3 \xi^{2}-2\right)+C\left(1+6 \xi+6 \xi^{2}+\xi^{4}\right)} \frac{6 m}{h_{B}}
$$

which leads to $\kappa=-\frac{6 m}{h_{B}} \frac{\xi}{1+\xi^{3}}$ in the case when $C \simeq 1$.

It is interesting to notice that the maximum curvature at given misfit is obtained for $\xi=1 / 2$, i.e. when the thickness of the A layer is half of the thickness of the B layer. Back to the general case, if $f(C, \xi)$ denotes the second numerical factor in the right-hand side of (8) at fixed contrast, one can compute the optimal thickness ratio in order to obtain a prescribed curvature. The general behavior of the numerical factor $f(C, \xi)$ is provided in Figure 5.

For example and considering the experimental control during epitaxial growth in the $\mathrm{InP} / \mathrm{InGaAs}$ material system, it is possible to grow a $25 \mathrm{~nm}$ thickness $\mathrm{Ga}_{0.15} \mathrm{In}_{0.85} P$ layer (the $A$ layer) at $1 \%$ tensile strain on top of a $50 \mathrm{~nm}$ thickness $\operatorname{InAs}_{0.35} P_{0.65}$ layer (the $B$ layer) at $1 \%$ compressive strain - with respect to InP lattice parameter. The previous results predict that the macroscopic curvature radius of the stress-free $75 \mathrm{~nm}$ bilayer structure GaIn $P / \operatorname{InAs} P$ will be $\simeq 2.7 \mu \mathrm{m}$.

We conclude this section with the following general remark: as shown by relation $(8) \kappa \simeq 1 / \rho \simeq \frac{8 m}{9 h_{B}}$ but in practical situations the thicknesses of layers $A$ and $B$ are limited by MBE technology. Increase of $m$ is possible by changing the composition of the two layers but this in turn may decrease the critical thickness of $2 \mathrm{~d}$ (planar) growth and raise the problem concerning the mechanical resistance of the bilayer after etching.

\subsection{The design problem}

As shown in the previous section, after etching, a free-stress bilayer material (obtained by the successive deposition of two materials with cubic symmetry and controlled thicknesses) will bend and the bending curvature tensor has the general expression $\boldsymbol{\kappa}=\kappa \boldsymbol{I}$ where $\kappa$ is given by (8). For completeness, we recall here that the curvature tensor used in the theory of linear elastic plates involves only the lower-order terms of the (geometrically exact) curvature tensor, i.e.

$$
\kappa \simeq-\left(\begin{array}{cc}
\frac{\partial^{2} w}{\partial x_{1}^{2}} & \frac{\partial^{2} w}{\partial x_{1} \partial x_{2}} \\
\frac{\partial^{2} w}{\partial x_{1} \partial x_{2}} & \frac{\partial^{2} w}{\partial x_{2}^{2}}
\end{array}\right) .
$$




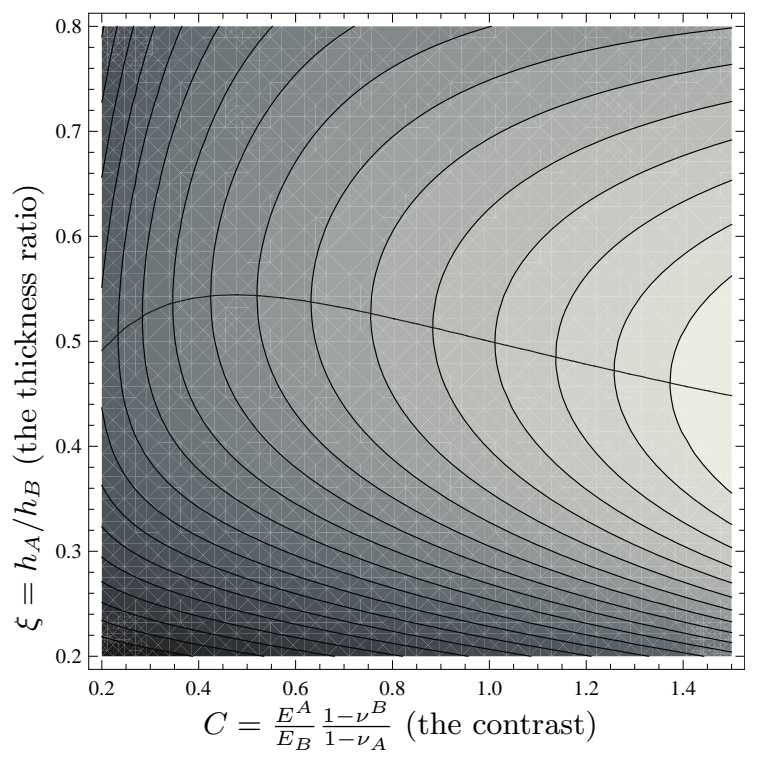

Figure 5. Values of the numerical factor $f(C, \xi)$ in (8). The continuous line give the thickness ratio (on the vertical axis) as a function of the contrast (horizontal axis) that provides the maximum curvature of the bilayer. As shown, the ratio thickness $\xi$ that leads to maximum curvature take values in the interval $(0.4,0.6)$ for various values of the contrast $C$.
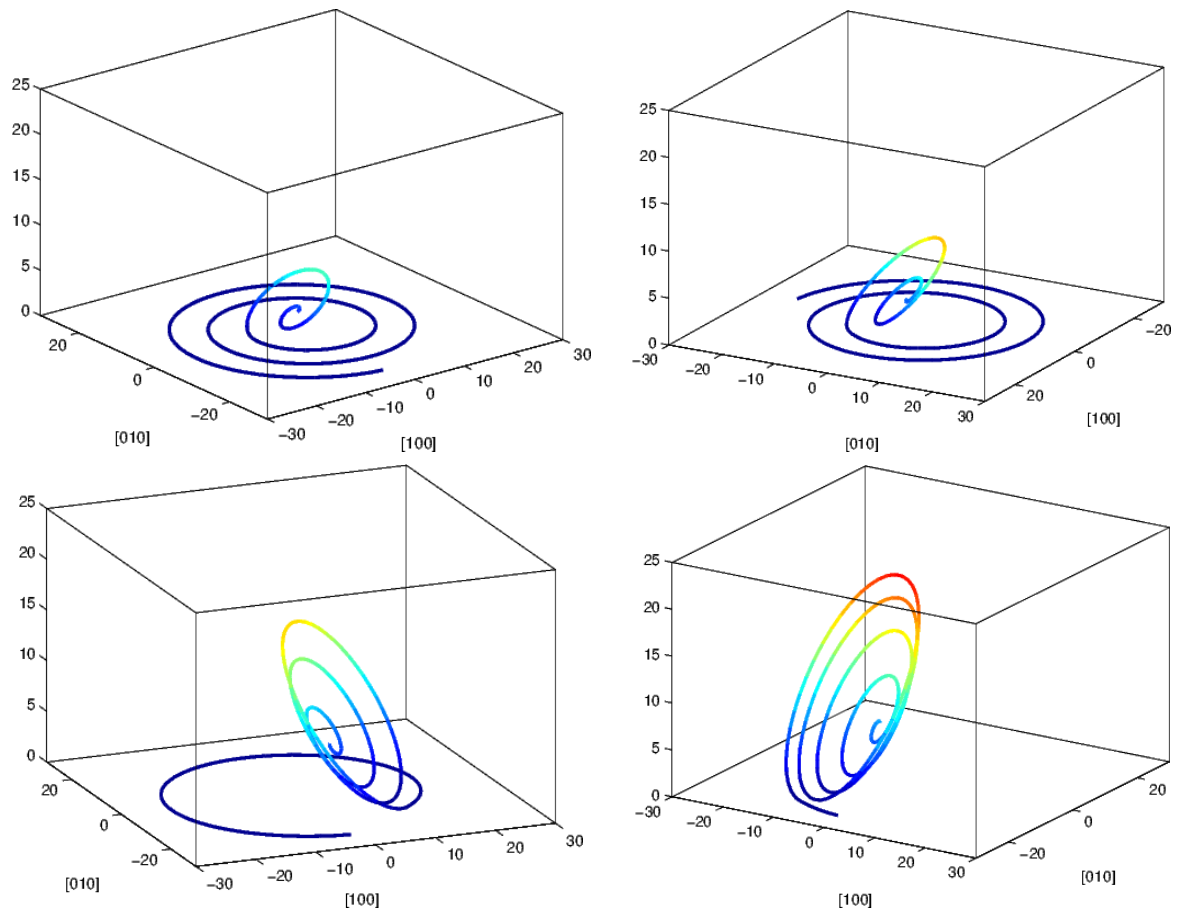

Figure 6. Four successive frames obtained by numerical simulation of the stress-relaxation folding problem for a generic spiral mask. The colors (in the online version) represent the elevation of different points form the mask plane due to stress-relaxation.

It is well-known that at this level of approximation the theory of linear elastic plates gives accurate results but it is unable to predict large deformations (as for example the folding of a long sheet) induced by finite displacements including rotations. To overcome this drawback we interpret the (experimentally confirmed) 
computed value of the curvature tensor in terms of exact geometric curvature* and address here the general design problem relating the form of a planar mask design and the shape of the three-dimensional object obtained after etching through the stress-relaxation process. For feasibility reasons we shall focus first on the folding problem of a given design mask. Then we shall address the unfolding problem, i.e., we look for a planar mask design which after folding will recover a fixed given three-dimensional shape.

The Figure 6 presents 4 successive frames induced by stress-relaxation of a spiral mask on a generic bilayer material. For simplicity the color on the graph represents the elevation of respective point with respect to the horizontal plane where the mask is designed. The perspective in different pictures are different to offer the best view for the three-dimensional object.

The Unfolding Problem The method used for the modeling of the folding problem allows to solve also the unfolding problem. Figure 7 presents 4 frames and the final mask design which after etching will create a spiral three-dimensional frame-wire on a sphere which is the basic object we look for. The numerical results are then implemented through an intermediate algorithm into the mask technology tool.

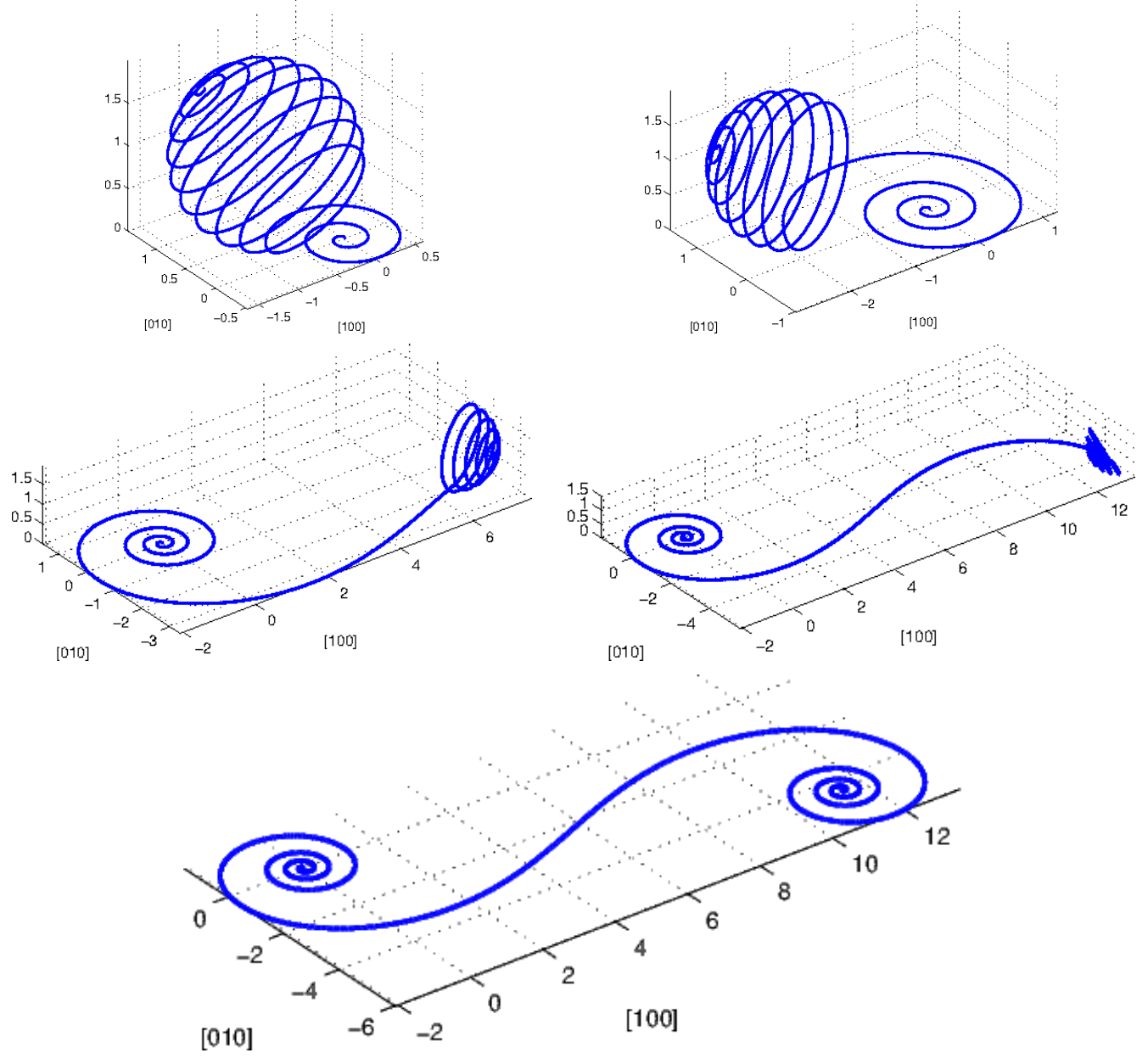

Figure 7. Five successive frames obtained by numerical simulation for the unfolding problem of a generic spherical frame. We notice that the size of the final two-dimensional domain is much larger that the initial size of the three dimensional object as indicated by the axes grid (same perspective for all frames).

\footnotetext{
${ }^{*}$ The details of the adjacent theory, computational method and associated results will be presented elsewhere.
} 


\section{PHOTON CAGE FABRICATION}

Fabrication of 3D closed microstructures consists in exploiting the process of elastic relaxation of pattern formed in pre-stressed multi-layer structures. The final shape of these objects are predetermined by the distribution of the deformations in the various semiconductor layers, imposed during their epitaxial growth, before their freestanding from the substrate by selective micromachining. The principal factor of control of the final shape of the objects is the gradient of deformation as explained in the previous section. Of course, the final shape of the 3D structures not only depends on strain gradient, but also on the micromachining parameters (crystallographic directions and etching kinetics).

Method for fabrication of free-standing 3D microstructures from strained $\mathrm{InP} / \mathrm{InGaAs}$ heterostructure was developed. The epitaxial structure consists of a $25 \mathrm{~nm}$ thick $\operatorname{In}(\mathrm{Ga}) \mathrm{P}-1 \%$ tensile - onto $50 \mathrm{~nm}$ thick $\operatorname{In}(\mathrm{As}) \mathrm{P}$ - 1\% compressive - strained bilayer on top of a 500 $\mathrm{nm}$ thick InGaAs lattice matched sacrificial layer grown on (100) InP substrate.

The microspiral formation was done by postgrowth e-beam lithography, vertical plasma transfer through the all $\mathrm{InP} / \mathrm{InGaAs}$ heterostructure and selective chemical underetching using $\mathrm{FeCl}_{3}$ for the InGaAs sacrificial layer. The mask pattern used for lithographic patterning contains 2 symmetric spirals tied together by a common central anchorage with diameters varying from 5 to $20 \mu m$ (see Figure 8 (left side)). The fabrication of the 3D closed microstructure based on the self-rolling up of the 2 spirals was carried out using the following steps: ebeam lithographic patterning on negative resist, $\mathrm{CH}_{4} / \mathrm{H}_{2}$ plasma etching to transfer the pattern to the epitaxial structure down to the sacrificial InGaAs. Then a timed control etch using $\mathrm{HF}: \mathrm{H}_{2} \mathrm{O}: \mathrm{H}_{2} \mathrm{O}_{2}$ was used to laterally remove InGaAs and release the InP-bilayer from the substrate for rolling. A final $\mathrm{CO}_{2}$ supercritical drying step was applied. Scanning electron microscopy (SEM) imaging was used to examine the topography and dimensions of the formed 3D microstructures (Fig. 8).
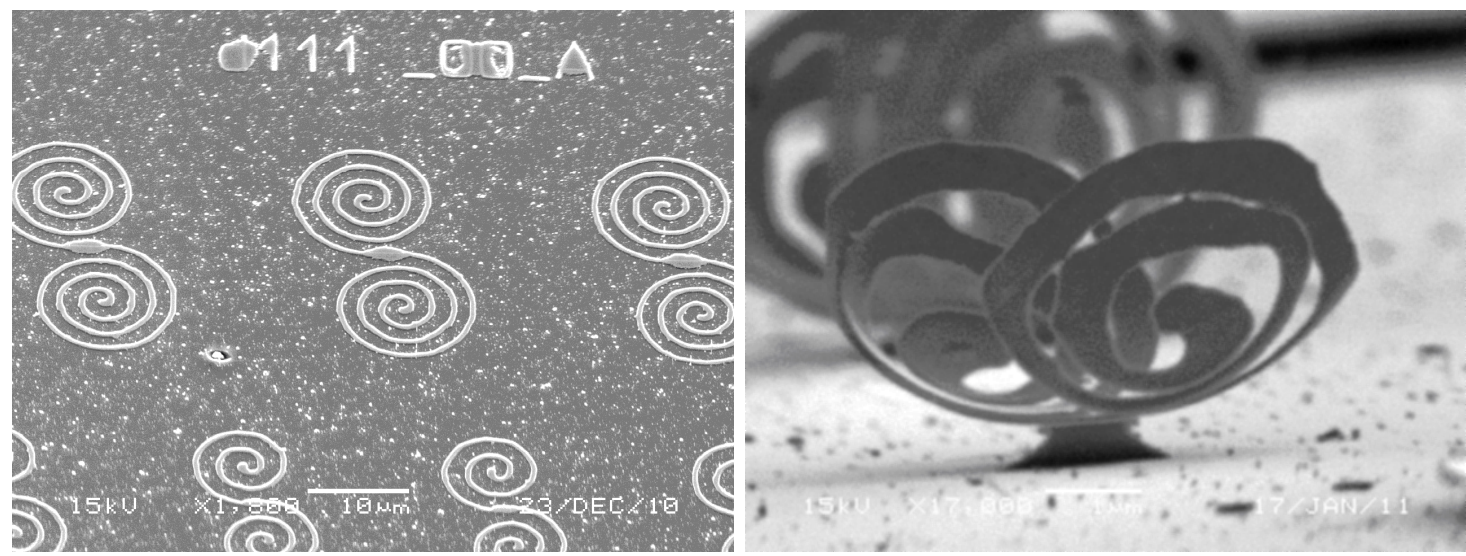

Figure 8. Optical image of the spirals with dimension from 5 to $20 \mu m$ (upper left part) SEM image of spirals with a diameter of $10 \mu \mathrm{m}$ after development (left). SEM image of the formed 3D spiral with diameter of $5 \mu \mathrm{m}$ (right).

\section{PERSPECTIVE AND CONCLUSIONS}

A spherical microcavity is demonstrated exhibiting a high confinement of light in its embedded air region. Additional electromagnetic simulations will be done to improve the 3D microresonator properties, especially in terms of modal volume and $\mathrm{Q}$-factor. We demonstrate controlled fabrication of spherical 3D microstructures in order to validate the design principles based on progressive relaxation of the elastic energy accounting for out-of-plane relaxation. We use the pre-stressed InGaP/InAsP bilayer films as a model system, which are grown with good accuracy by molecular beam epitaxy. Experimental works are now under progress considering the unfolding problem, allowing the fabrication of a real spherical microcavity with constant filling factor. We believe that the micro-nanomechanical architecture of strained bilayer thin films can be now considered as very versatile and powerful for fabrication of various original 3D microstructures. 


\section{Acknowledgement}

Authors are gratefully acknowledging technological staffs from Nanolyon platform. Region Rhne-Alpes is also acknowledged for its financial support through the $\mathrm{PhD}$ grant of C. Sieutat.

\section{REFERENCES}

[1] John, S., "Strong localization of photons in certain disordered dielectric superlattices," Phys. Rev. Lett. 58, 2486-2489 (Jun 1987).

[2] Nelson, E. C. and Braun, P. V., "Photonic crystals: Photons and electrons confined," Nature Photonics 2, 650-651 (Nov. 2008).

[3] Yablonovitch, E., "Inhibited spontaneous emission in solid-state physics and electronics," Phys. Rev. Lett. 58, 2059-2062 (May 1987).

[4] Mekis, A., Chen, J. C., Kurland, I., Fan, S., Villeneuve, P. R., and Joannopoulos, J. D., "High transmission through sharp bends in photonic crystal waveguides," Phys. Rev. Lett. 77, 3787-3790 (Oct 1996).

[5] Inoue, K. and Ohtaka, K., "Photonic crystals: physics, fabrication, and applications," Springer Series on Optical Sciences 94 (2004).

[6] Prinz, V., Seleznev, V., Gutakovsky, A., Chehovskiy, A., Preobrazhenskii, V., Putyato, M., and Gavrilova, T., "Free-standing and overgrown InGaAs/GaAs nanotubes, nanohelices and their arrays," PHYSICA E 6, 828-831 (FEB 2000).

[7] Li, X., "Strain induced semiconductor nanotubes: from formation process to device applications," Journal of Physics D: Applied Physics 41, 193001+ (Oct. 2008).

[8] Letartre, X., Mouette, J., Leclercq, J. L., Romeo, P. R., Seassal, C., and Viktorovitch, P., "Switching devices with spatial and spectral resolution combining photonic crystal and moems structures," J. Lightwave Technol. 21, 1691 (Jul 2003).

[9] Sieutat, C., Leclercq, J., Letartre, X., Callard, S., Gendry, M., Grenet, G., Naji, K., Regreny, P., RojoRomeo, P., Viktorovitch, P., Beaudin, G., Cloutier, M., Drouin, D., and Aimez, V., "3d harnessing of light with photon cage," Proceedings of SPIE - The International Society for Optical Engineering 7712 (2010).

[10] Boutami, S., Benbakir, B., Letartre, X., Leclercq, J. L., Regreny, P., and Viktorovitch, P., "Ultimate vertical Fabry-Perot cavity based on single-layer photonic crystal mirrors," Opt. Express 15(19), 12443-12449 (2007). 